\title{
Powerful terahertz free electron lasers with hybrid Bragg reflectors
}

\author{
N. S. Ginzburg, A. M. Malkin, ${ }^{*}$ N. Yu. Peskov, A. S. Sergeev, V. Yu. Zaslavsky, and I. V. Zotova \\ Institute of Applied Physics RAS, Nizhny Novgorod, Russia \\ (Received 25 December 2010; published 7 April 2011)
}

\begin{abstract}
For the electron devices with oversized interaction space, the Bragg structures of different types are effective instruments for controlling and redistribution of the electromagnetic energy fluxes to provide a spatial coherence of radiation. In this paper a powerful long-pulse free-electron laser of the terahertz waveband is studied in which an advanced Bragg structure based on the coupling between the propagating waves and the cutoff mode is used as an upstream reflector in the two-mirror resonator. In the case of planar geometry, this structure provides effective mode selection over the "narrow" transverse coordinate directed between the plates. Under the assumption that the system is open over a second "wide" coordinate, the diffraction effects are sufficient for synchronization of radiation from a sheet electron beam over this direction. An alternative way of radiation synchronization for a case of more extended electron beam by exploiting a 2D Bragg structure as a downstream reflector is discussed.
\end{abstract}

DOI: 10.1103/PhysRevSTAB.14.042001

PACS numbers: 41.60.Cr, 42.25.Fx

\section{INTRODUCTION}

Distributed feedback involving periodical Bragg structures is widely used in generators of coherent radiation based on principles of both classical and quantum electronics. Correspondingly, the frequency range in which the oscillators of this type effectively operate includes millimeter and optical (infrared) bands [1-4]. In [5-7] a new modification of Bragg structures was suggested that can be applied to the terahertz band free-electron lasers. The specific feature of this advanced Bragg structure (ABS) is the coupling between propagating and cutoff modes. The advantage of $\mathrm{ABS}$ along with the possibility of mode selection in an oversized electrodynamical system is their compatibility with intense electron beam transport channels that are beneficial for development of long (microsecond) pulse $\mathrm{THz}$ free-electron lasers (FELs) of megawatt power level.

In Fig. 1(a) the scheme of a planar THz FEL with a hybrid two-mirror cavity consisting of an ABS as an upstream mirror and a conventional Bragg structure as a downstream mirror is shown. Indicated structures are formed by the sections of a planar waveguide with shallow sine corrugation of the surface,

$$
b(z)=b_{0}+b_{j} \cos \left(\bar{h}_{j} z\right),
$$

where $b_{0}$ is the width of the gap between the plates, $b_{j}$ are the amplitudes of corrugation, $\bar{h}_{j}=2 \pi / d_{j}, d_{j}$ are their periods. Here index $j=1$ refers to the parameters of the

\footnotetext{
*malkin@appl.sci-nnov.ru
}

Published by American Physical Society under the terms of the Creative Commons Attribution 3.0 License. Further distribution of this work must maintain attribution to the author(s) and the published article's title, journal citation, and DOI. upstream structure and the index $j=2$ to the parameters of the downstream one.

In a conventional downstream Bragg reflector, the coupling between the two paraxial wave beams propagating in opposite directions is employed when the Bragg resonance condition is satisfied,

$$
\bar{h}_{2} \approx 2 h,
$$

where $h$ is the longitudinal wave number of forward and backward waves.

The peculiarity of the advanced Bragg structure is the absence of direct coupling between the counterpropagating
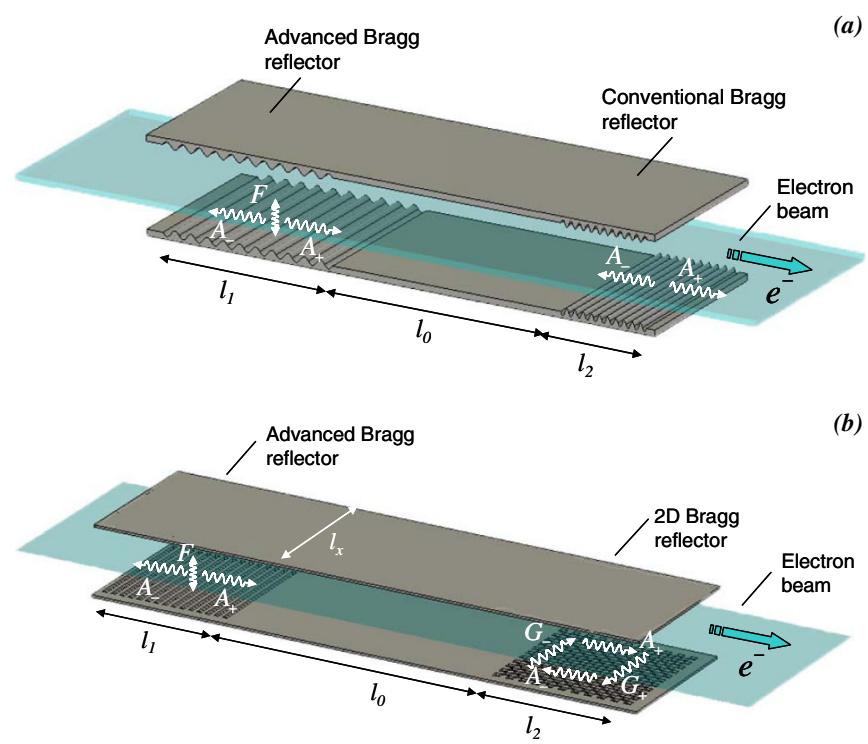

FIG. 1. The schemes of planar FEL with advanced upstream Bragg reflector. As downstream reflectors can be used either conventional (a) or two-dimensional (b) Bragg structures. Arrows mark the directions of wave-beam propagation. 
paraxial waves. Instead, paraxial waves are coupled with a cutoff mode [6,7] under the following Bragg resonance condition:

$$
\bar{h}_{1} \approx h \text {. }
$$

Obviously the period of the advanced Bragg structure is 2 times larger than for the conventional one. Scattering into the $\mathrm{TM}_{s}$ mode possessing the cutoff frequency $\omega_{c}=\pi s c / b_{0}$ occurs when the gap between the plates and the corrugation period is bound by the condition

$$
b_{0} \approx s d_{1} / 2 \text {, }
$$

where $s$ is integer.

In Fig. 1(b) a more sophisticated scheme of Bragg FEL is presented. Here a 2D Bragg structure is used as downstream reflector $[8,9]$ which allows one to increase the system size over the open $x$ coordinate. This structure is formed by the plates with double-periodical corrugation:

$$
b(z)=b_{0}+\frac{b_{2 \mathrm{D}}}{2}\left\{\cos \left[\bar{h}_{2 \mathrm{D}}(z-x)\right]+\cos \left[\bar{h}_{2 \mathrm{D}}(z+x)\right]\right\} .
$$

The Bragg condition takes the form

$$
\bar{h}_{2 \mathrm{D}} \approx \sqrt{2} h .
$$

The 2D Bragg structure provides coupling of wave beams propagating in the $\pm z$ directions with additional wave beams propagating in the transverse $\pm x$ directions that synchronize radiation of the large-size sheet electron beams.

Obviously the complete analysis of planar THz FEL with overmoded Bragg structures described above should include the solutions of $3 \mathrm{D}$ problems. In the present paper we reduce under certain conditions this problem to several simplified 2D models. In Sec. II we investigate the nonlinear dynamics of FEL with an ABS as an upstream mirror and a conventional Bragg structure as a downstream mirror [Fig. 1(a)]. Unlike previous considerations [5,6], in this paper the structure of paraxial wave beams over the $y$ axis is not assumed to be fixed and presents a sum of modes of regular planar waveguide. This allows us to be more accurate in determining the maximum acceptable oversize factor (ratio between the gap $b_{0}$ and the wavelength $\lambda$ ) that still maintains the coherence of radiation over the $y$ axis. Then under the assumption that a planar system is open in the $x$ direction, we study the formation of spatial radiation structure along this coordinate. We show that for a sheet electron beam of finite width the optical guiding effects [10-12] are sufficient for spatial synchronization of radiation while the corresponding Fresnel condition [see (35) below] is satisfied. In Sec. III we study an FEL with hybrid Bragg resonator which combines an advanced upstream and two-dimensional downstream mirrors [Fig. 1(b)] that allows further extension of system size over the $x$ direction.

\section{FEL WITH ADVANCED UPSTREAM AND CONVENTIONAL DOWNSTREAM BRAGG REFLECTORS}

\section{A. Formation of wave structure over $y$ coordinate}

First we consider a 2D model in the assumption that the planar waveguide is infinite along the "wide" transverse coordinate $x$ [Fig. 1(a)]. When the Bragg scattering condition (3) is satisfied, the field in the advanced Bragg structure can be presented as a sum of the two quasioptical wave beams propagating in opposite directions over the $z$ axis:

$$
\vec{H}=\vec{x}_{0} \operatorname{Re}\left\lfloor A_{+}(z, y, t) e^{i(\omega t-h z)}+A_{-}(z, y, t) e^{i(\omega t+h z)}\right\rfloor
$$

and a cutoff $\mathrm{TM}_{s}$ mode

$$
\vec{H}=\vec{x}_{0} \operatorname{Re}\left\lfloor F(z, t) \sin \left(h_{\perp} y\right) e^{i \omega t}\right\rfloor .
$$

Here $A_{ \pm}(z, y, t)$ and $F(z, t)$ are the slowly varying amplitudes; the carrier frequency is chosen to be equal to the cutoff frequency: $\omega=\omega_{c}$. The electric fields are given by the expression

$$
\vec{E}=-i(c / \omega) \operatorname{rot} \vec{H}
$$

In an FEL the electron beam wiggling in the undulator field amplifies the comoving wave beam $A_{+}$under the resonance condition

$$
\omega-h V_{0} \approx h_{u} V_{0},
$$

where $h_{u}=2 \pi / d_{u}$ and $d_{u}$ is the undulator period. The synchronous wave $A_{+}$scatters into the backward wave $A_{-}$via the excitation of the cutoff mode $F$ on a Bragg structure. As a result the coupling between the cutoff and propagating wave beams makes it possible to combine the mode selection mechanisms used in gyrotrons and orotrons $[13,14]$ with Doppler frequency up-conversion typical for FEL.

Nonstationary equations for the amplitudes of coupled waves can be presented in the form [15]

$$
\begin{aligned}
\frac{\partial a_{+}}{\partial Z}+\frac{\partial a_{+}}{\partial \tau}+i \frac{\partial^{2} a_{+}}{\partial Y^{2}} & =i \frac{\alpha_{1} B}{2} f[\delta(Y)+\delta(Y-B)]+\chi(Y) J \\
-\frac{\partial a_{-}}{\partial Z}+\frac{\partial a_{-}}{\partial \tau}+i \frac{\partial^{2} a_{-}}{\partial Y^{2}}= & i \frac{\alpha_{1} B}{2} f[\delta(Y)+\delta(Y-B)] \\
\frac{\partial f}{\partial \tau}+\frac{i C}{2} \frac{\partial^{2} f}{\partial Z^{2}}+\sigma f & =i \alpha_{1}\left[\left.\left(a_{+}+a_{-}\right)\right|_{Y=0}\right. \\
& \left.+\left.\left(a_{+}+a_{-}\right)\right|_{Y=B}\right]
\end{aligned}
$$

where $\delta(Y)$ is the delta function. Deriving Eqs. (9) we used the concept of surface magnetic current developed in $[16,17]$. Here we introduce the following normalized variables and parameters:

$$
\begin{aligned}
& Z=C \frac{\omega_{c}}{c} z, \quad Y=\sqrt{2 C} \frac{\omega_{c}}{c} y, \quad \tau=C \omega_{c} t, \quad a_{ \pm}=\frac{e \mu K A_{ \pm}}{2 m c \omega_{c} \gamma_{0}^{2} C^{2}}, \\
& f=\frac{e \mu K F}{2 \sqrt{2} m c \omega_{c} \gamma_{0}^{2} C^{2}} C=\left(\frac{e I_{0} \mu K^{2} \lambda^{2}}{8 \pi m c^{3} \gamma_{0}^{3} b_{0}}\right)^{1 / 3}
\end{aligned}
$$


is the gain parameter, $I_{0}$ is the linear current density, $K=e H_{u} / h_{u} m c^{2}, H_{u}$ is the undulator field amplitude, $\mu \approx \gamma_{0}^{-2}\left(1+K^{2}\right)$ is the electron bunching parameter, $\gamma_{0}=\left(1-\beta_{0}^{2}\right)^{-1 / 2}, \quad \lambda=2 \pi c / \omega_{c}, \quad \sigma=\nu / C b_{0}$ is the Ohmic losses parameter for the cutoff mode, $\nu$ is the skin depth (Ohmic losses for propagating waves $a_{ \pm}$are negligibly small), $\alpha_{1}=b_{1} / C b_{0} \sqrt{2}$ is the waves coupling coefficient. Function $\chi(Y)$ describes the distribution of the electron current over the $y$ axis. We normalize it as $\int_{0}^{B} \chi(Y) d Y=B$, where $B=\sqrt{2 C} \omega_{c} b_{0} / c$ is the normalized gap between plates. The excitation factor for the synchronous wave (the rf current),

$$
J=1 / \pi \int_{0}^{2 \pi} e^{-i \theta} d \theta_{0}
$$

can be found from the averaged electron motion equations

$$
\left(\frac{\partial}{\partial \tau}+\beta_{0}^{-1} \frac{\partial}{\partial Z}\right)^{2} \theta=\operatorname{Re}\left(a_{+} e^{i \theta}\right)
$$

with boundary conditions

$$
\left.\theta\right|_{Z=0}=\theta_{0} \in[0,2 \pi),\left.\quad\left(\frac{\partial}{\partial \tau}+\beta_{0}^{-1} \frac{\partial}{\partial Z}\right) \theta\right|_{Z=0}=-\Delta .
$$

In the conventional output Bragg mirror, the direct coupling between forward and backward propagating waves takes place which can be described by the equations

$$
\begin{aligned}
\frac{\partial a_{+}}{\partial Z}+\frac{\partial a_{+}}{\partial \tau}+i \frac{\partial^{2} a_{+}}{\partial Y^{2}}= & i \frac{\alpha_{2} B}{2} a_{-}[\delta(Y)+\delta(Y-B)] \\
& +\chi(Y) J \\
-\frac{\partial a_{-}}{\partial Z}+\frac{\partial a_{-}}{\partial \tau}+i \frac{\partial^{2} a_{-}}{\partial Y^{2}}= & i \frac{\alpha_{2} B}{2} a_{+}[\delta(Y)+\delta(Y-B)],
\end{aligned}
$$

where $\alpha_{2}=b_{2} / C b_{0}$ is the coupling coefficient in the conventional Bragg structure.

In the regular resonator section, the amplification of synchronous wave is described by the equation

$$
\frac{\partial a_{+}}{\partial Z}+\frac{\partial a_{+}}{\partial \tau}+i \frac{\partial^{2} a_{+}}{\partial Y^{2}}=\chi(Y) J,
$$

together with electron motion equations (10).

Taking into account the boundary conditions on the metal surfaces

$$
\left.\frac{\partial a_{ \pm}}{\partial Y}\right|_{Y=0, B}=0,
$$

we expand the wave beams into Fourier series

$$
a_{ \pm}=\sum_{n=0}^{\infty} a_{ \pm}^{n}(Z, \tau) \cos \left(\frac{n \pi Y}{B}\right) .
$$

Each term of (15) characterized by the $n$ index can be treated as a mode of a regular planar waveguide with an amplitude $a_{ \pm}^{n}$. After the mode expansion the equations (9) and (12) describing the electron-wave interaction can be transformed to the following form:

$$
\begin{aligned}
\frac{\partial a_{+}^{n}}{\partial Z}+\frac{\partial a_{+}^{n}}{\partial \tau}-i S n^{2} a_{+}^{n} & =\frac{2 i \alpha_{1} f}{1+\delta_{0 n}}+J_{n} \\
-\frac{\partial a_{-}^{n}}{\partial Z}+\frac{\partial a_{-}^{n}}{\partial \tau}-i S n^{2} a_{-}^{n} & =\frac{2 i \alpha_{1} f}{1+\delta_{0 n}} \\
\frac{i C}{2} \frac{\partial^{2} f}{\partial Z^{2}}+\frac{\partial f}{\partial \tau}+\sigma f & =i \alpha_{1} \sum_{n=0}^{\infty}\left(a_{+}^{n}+a_{-}^{n}\right)
\end{aligned}
$$

in the input advanced Bragg mirror, and

$$
\begin{aligned}
\frac{\partial a_{+}^{n}}{\partial Z}+\frac{\partial a_{+}^{n}}{\partial \tau}-i S n^{2} a_{+}^{n} & =\sum_{m=0}^{\infty} \frac{2 i \alpha_{2} a_{-}^{m}}{1+\delta_{0 n}}+J_{n} \\
-\frac{\partial a_{-}^{n}}{\partial Z}+\frac{\partial a_{-}^{n}}{\partial \tau}-i S n^{2} a_{-}^{n} & =\sum_{m=0}^{\infty} \frac{2 i \alpha_{2} a_{+}^{m}}{1+\delta_{0 n}}
\end{aligned}
$$

in the output conventional Bragg mirror. Here $S=\pi^{2} / B^{2}$, $\delta_{0 n}$ is the Kronecker delta,

$$
J_{n}=\frac{2}{1+\delta_{0 n}} \frac{1}{B} \int_{0}^{B} J \cos \left(\frac{n \pi Y}{B}\right) d Y .
$$

Note that deriving (18) we assumed for simplicity that the electron beam is uniformly distributed between the waveguide walls: $\chi(Y)=1$.

In the limit case of a small cross-section waveguide

$$
S \gg 1, \quad B \ll \pi,
$$

one can neglect the reradiation into the higher modes and Eqs. (16) and (17) reduce to the form used in our previous paper [5,6], where we assumed that the transverse structure of propagating waves coincides with that of the TEM modes of the regular planar waveguide. Under the assumption of fixed transverse field structure, the interaction in the advanced Bragg mirror can be described by the equations

$$
\begin{gathered}
\frac{\partial a_{+}^{0}}{\partial Z}+\frac{\partial a_{+}^{0}}{\partial \tau}=i \alpha_{1} f+J \quad-\frac{\partial a_{-}^{0}}{\partial Z}+\frac{\partial a_{-}^{0}}{\partial \tau}=i \alpha_{1} f \\
\frac{i C}{2} \frac{\partial^{2} f}{\partial Z^{2}}+\frac{\partial f}{\partial \tau}+\sigma f=i \alpha_{1}\left(a_{+}^{0}+a_{-}^{0}\right)
\end{gathered}
$$

and in the conventional one by the equations

$$
\frac{\partial a_{+}^{0}}{\partial Z}+\frac{\partial a_{+}^{0}}{\partial \tau}=i \alpha_{2} a_{-}^{0}+J \quad-\frac{\partial a_{-}^{0}}{\partial Z}+\frac{\partial a_{-}^{0}}{\partial \tau}=i \alpha_{2} a_{+}^{0} .
$$

Formulating the boundary conditions for Eqs. (9) and (12), one may assume that the energy fluxes from outside are absent and the amplitudes of propagating waves on the corresponding boundaries turn to zero:

$$
a_{+}(Z=0)=0, \quad a_{-}(Z=L)=0,
$$

where $L=L_{1}+L_{0}+L_{2}$ is the total normalized length of the system, $L_{j}=C h l_{j}$. Besides we assume the continuity of the wave amplitude on the boundaries between the different sections.

For a quasicutoff mode described by the parabolic equation (9) on the edges of the input mirror, we apply 
the well-known in the gyrotron theory $[18,19]$ radiation boundary conditions:

$$
\left.f\left(Z=0, L_{1}\right) \mp \sqrt{\frac{C}{\pi i}} \int_{0}^{\tau} \frac{e^{-\sigma\left(\tau-\tau^{\prime}\right)}}{\sqrt{\tau-\tau^{\prime}}} \frac{\partial f\left(\tau^{\prime}\right)}{\partial Z}\right|_{Z=0, L_{1}} d \tau^{\prime}=0 .
$$

In the steady-state oscillation regime, $a_{ \pm}, f \sim \exp (i \Omega \tau)$ $\left[\Omega=\left(\omega-\omega_{c}\right) / C \omega_{c}\right.$ is the detuning of the generated frequency from the carrier frequency], the energy conservation law can be obtained from the equations (9)

$$
\hat{\eta} B=P_{+}+P_{-}+P_{\mathrm{dif}}^{z}+P_{\mathrm{ohm}},
$$

where

$$
\begin{aligned}
& \eta=\frac{C}{\mu\left(1-\gamma_{0}^{-1}\right)} \hat{\eta}, \\
& \hat{\eta}=\left.\frac{1}{2 \pi B} \int_{0}^{B} \int_{0}^{2 \pi}\left(\frac{\partial \theta}{\partial Z}+\Delta\right)\right|_{Z=L} d \theta_{0} d Y
\end{aligned}
$$

is the electron efficiency,

$$
P_{+}=\frac{1}{4} \int_{0}^{B}\left|a_{+}\right|_{Z=L}^{2} d Y, \quad P_{-}=\frac{1}{4} \int_{0}^{B}\left|a_{-}\right|_{Z=0}^{2} d Y
$$

are the normalized power densities carried by paraxial wave beams,

$$
\begin{aligned}
P_{\mathrm{dif}}^{z} & =\frac{C B}{4} \operatorname{Im}\left(\left.f \frac{\partial f^{*}}{\partial Z}\right|_{Z=L}+\left.f^{*} \frac{\partial f}{\partial Z}\right|_{Z=0}\right), \\
P_{\mathrm{ohm}} & =\frac{\sigma B}{2} \int_{0}^{L}|f|^{2} d Z
\end{aligned}
$$

are the diffraction and Ohmic power losses of the quasicutoff mode, respectively. Gross output power is proportional to $P_{+}$, while all the other terms of (23) correspond to various channels of energy loss. For a numerical estimation of the total power we introduce

$$
\Gamma=\frac{P_{+}}{\hat{\eta} B}
$$

defined as a ratio between the gross output power and the total power radiated from the electron beam.

We simulate the nonlinear dynamics of an FEL based on Eqs. (10), (16), and (17) for the operating frequency $f=1 \mathrm{THz}$. We took the particles energy to be $5.5 \mathrm{MeV}$, the undulator period $4 \mathrm{~cm}$, and the undulator field amplitude $H_{u}=2.8 \mathrm{kOe}$. In this case for a sheet electron beam with injection current density $130 \mathrm{~A} / \mathrm{cm}^{2}$, the gain parameter is $C \approx 1.4 \times 10^{-4}$. Lengths of the input and the output Bragg reflectors were taken $l_{1,2}=13.5 \mathrm{~cm}$, corrugation depths $2 b_{1}=5 \mu \mathrm{m}$ and $2 b_{2}=1.5 \mu \mathrm{m}$, and the periods $d_{1}=$ $0.03 \mathrm{~cm}$ and $d_{2}=0.015 \mathrm{~cm}$; the length of the regular section was taken $l_{0}=150 \mathrm{~cm}$. For a waveguide walls made of copper the skin depth is $\delta \approx 0.01 \mu \mathrm{m}$.

Results of the simulations of FEL operation are presented in Fig. 2 at normalized values $B=1$ and $B=2$, that correspond to the gap between the resonator plates equal to 10 and $20 \lambda$. The current density was assumed to be constant and the electron beam to uniformly fill the cavity over the $y$ coordinate. Thus, the total injection current was larger for a larger gap while the gain parameter stayed constant. The Ohmic losses parameter was in the first case equal to $\sigma=0.18$ and in the second one to $\sigma=0.09$. At constant corrugation depth the normalized coupling coefficients $\alpha_{1,2}$ decrease with increasing of the gap.

Simulation demonstrates that at the chosen parameters the considered FEL scheme provides the establishment of the single-frequency steady-state regime (Fig. 2). Spatial distributions of the partial waves amplitudes $\hat{a}_{ \pm}$in the steady-state regime presented in Fig. 3 show that the output
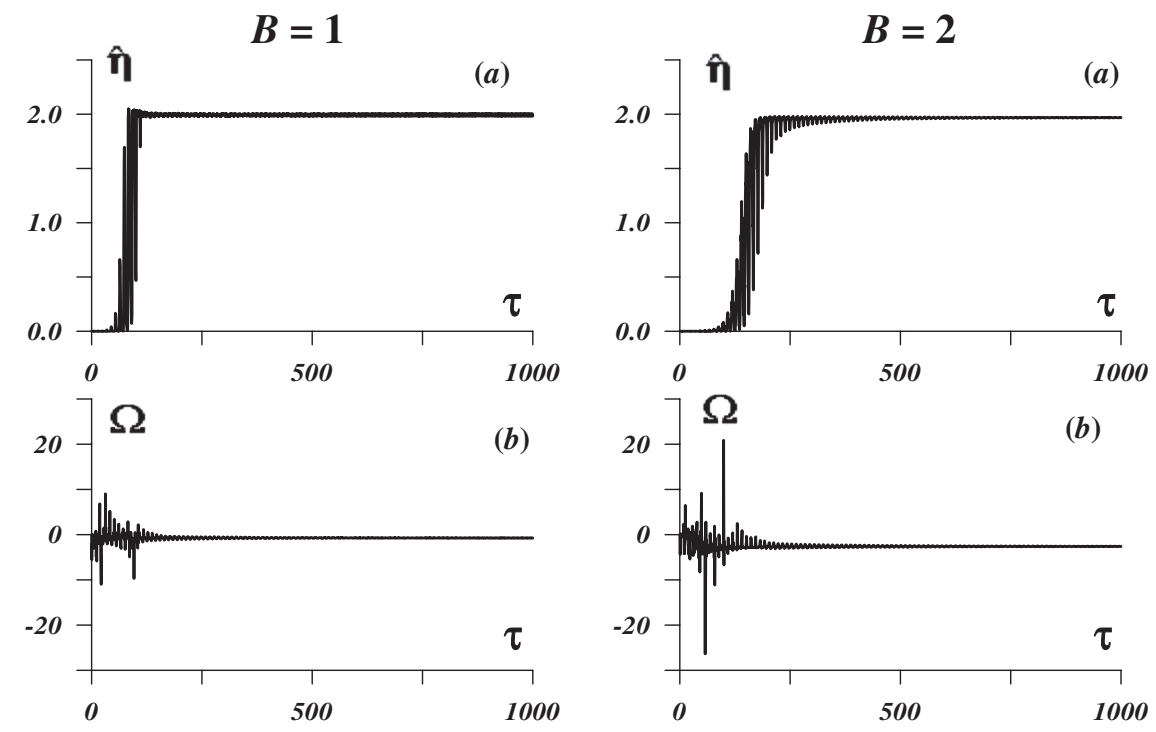

FIG. 2. Establishment of steady-state oscillations at various values of the gap parameter: $B=1$ (left, $\alpha_{1}=4, \alpha_{2}=1$ ), $B=2$ (right, $\left.\alpha_{1}=2, \alpha_{2}=0.5\right)$. 

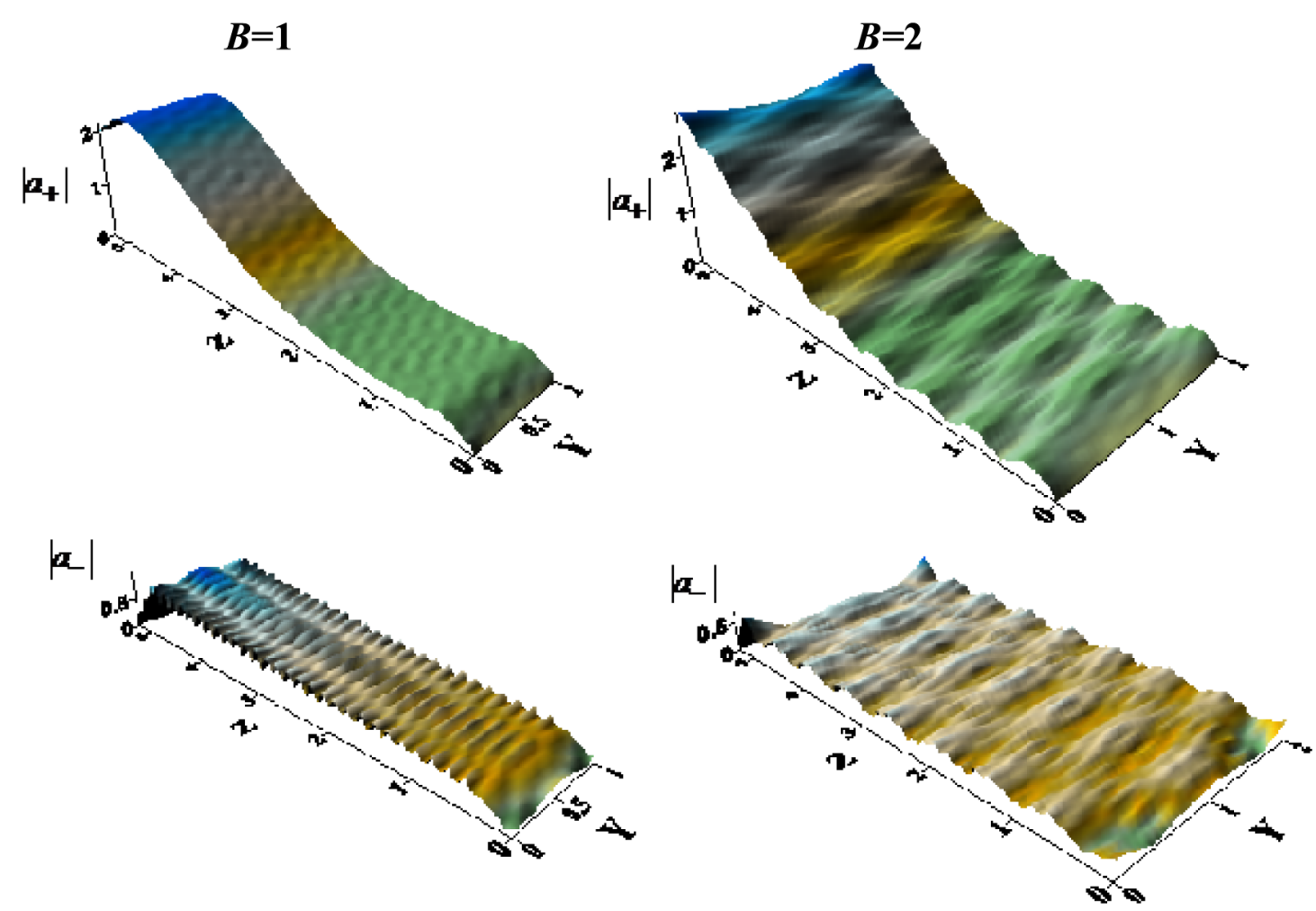

FIG. 3. Spatial structures of the propagating partial waves $a_{ \pm}$in the steady-state regime for the same parameters as in Fig. $2: B=1$ (left), $B=2$ (right).

radiation possesses rather high spatial homogeneity over the $y$ axis and its structure is close to the TEM mode. Because of the input advanced Bragg reflector this regime is stable to the changing of the electron beam parameters. In Fig. 4 we show that variation of the electron synchronism detuning $\Delta$ leads to the smooth changing of the radiation frequency. At $B=1 \quad\left(b_{0}=10 \lambda\right)$ there are no frequency jumps and at $B=2\left(b_{0}=20 \lambda\right)$ there is a

$$
B=1
$$
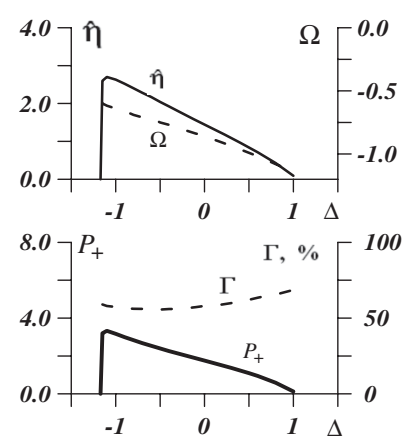

(a)

$$
B=2
$$
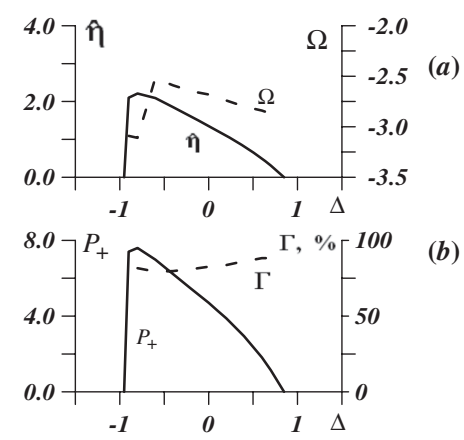

(b)

FIG. 4. Dependence of the steady-state regime characteristics on the electron synchronism detuning parameter $\Delta$ for the normalized gap $B=1$ (left), $B=2$ (right) at the same parameters as in Fig. 2: (a) normalized efficiency $\hat{\eta}$ and the electron frequency shift $\Omega$; (b) normalized output power $P$ and its a ratio to the total power radiated from the beam $\Gamma$.

single-frequency jump near the boundary of the oscillations band. Admissible detuning spread $\Delta \sim 1$ corresponds to the energy spread $\delta \gamma / \gamma \sim C \gamma^{2} \sim 1 \%$.

Analysis of longitudinal field distribution shows (Fig. 5) that at the simulation parameters the main amplification of radiation by the electron beam takes place in the regular section of the waveguide after the input advanced Bragg mirror. The output traditional Bragg mirror provides relatively small (up to $25 \%$ power) reflection in the backward wave. Because of this the amplitude of the quasicutoff mode $\hat{f}$ excited in the input mirror is relatively small and so are the diffraction and Ohmic losses associated with this mode. The Ohmic losses value obviously depends on the gap between the plates. At the gap of $10 \lambda$ Ohmic losses reach $50 \%(\Gamma=50 \%)$ of the power radiated by the beam and for $20 \lambda$ gap these losses decrease down to $20 \%(\Gamma=80 \%)$ [Figs. 4(a) and 4(b)].

One should note that for a relatively small gap between plates the characteristics of an FEL under consideration can be described within the three coupled waves model used in our previous paper [5,6]. In Fig. 5 the longitudinal distributions of the fields are shown obtained from the full (10), (16), and (17) and the simplified model (10), (19), and (20). One can see that at $B=1\left(b_{0}=10 \lambda\right)$ the results are quite similar, while for the larger oversize factors the FEL operation should be simulated by using the full "multiwave" model developed in this paper. This model allows one to estimate the limits of the oversize factors $b_{0} / \lambda$ 

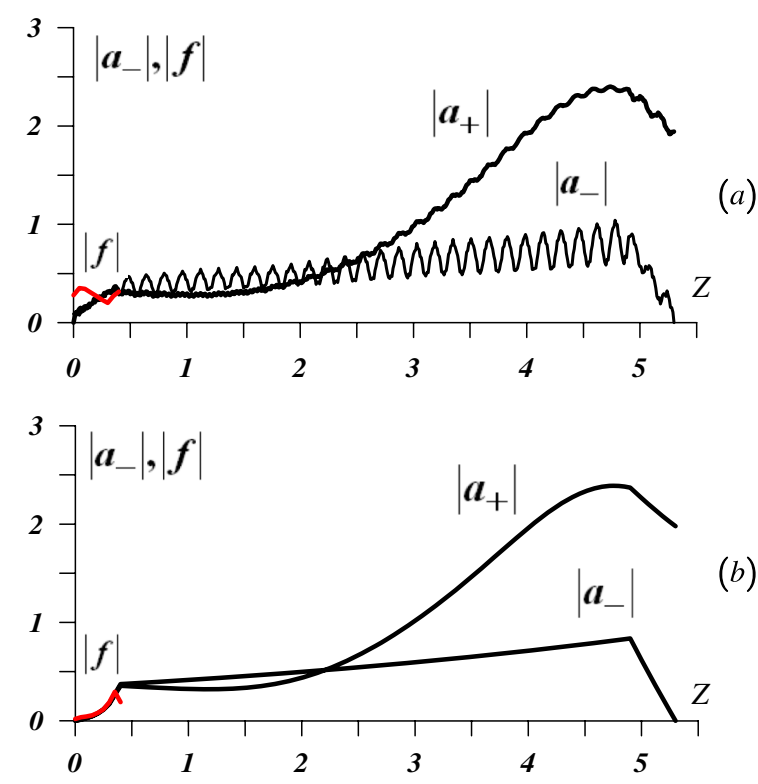

FIG. 5. Longitudinal distributions of partial waves $a_{ \pm}$and $f$ in the steady-state regime along the resonator axis $Y=B / 2$ at the moderate oversize factor $B=1$ (a) and similar curves obtained using the simplified model (19) and (20) with the fixed transverse structures of propagating waves (b) $\left(L_{1,2}=0.4, L_{0}=4\right.$, $\left.\alpha_{1}=4, \alpha_{2}=1, \Delta=-0.5, C \approx 1.4 \times 10^{-4}\right)$.

because the increasing of the gap leads to the complication of the mode content in output radiation. Spatial profiles of partial wave beams in the steady-state oscillation regime are presented in Fig. 6 at large oversize factor $B=$ $4\left(b_{0}=40 \lambda\right)$. In this case the mode expansion of output radiation comprises a large number of modes of a regular waveguide (Fig. 6).

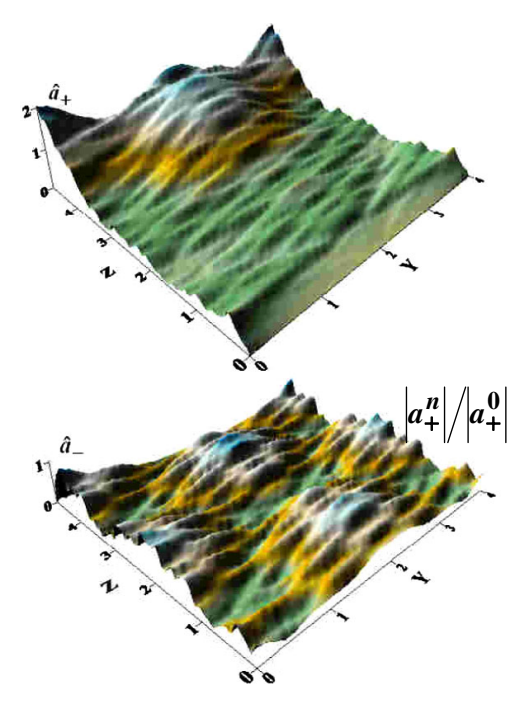

(b)

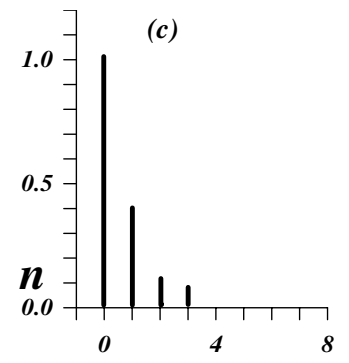

FIG. 6. Spatial distributions of partial wave fields $a_{ \pm}(Y, Z)$ $[(a),(b)]$ and the expansion of the output wave beam into series of the regular waveguide modes (c) in the steady-state regime $\left(B=4, L_{1,2}=0.4, L_{0}=4, \alpha_{1}=1, \alpha_{2}=0.5, \Delta=-1\right)$.
Thus, the suggested scheme of THz FEL allows one to provide the conditions of self-excitation, effective energy extraction, and spatial coherence of radiation at the oversize factors up to 10-20 wavelengths which is sufficient for the formation of transportation channel for the intense sheet beam. Simulation shows that at $b_{0}=20 \lambda$ up to $80 \%$ of radiated power is extracted from the interaction space with forward wave $\hat{a}_{+}$. At the electron efficiency of $\eta \sim 2 \%$ power density is about $6.5 \mathrm{MW} / \mathrm{cm}$. Establishment time of oscillations reaches $300 \mathrm{~ns}$.

\section{B. Formation of the wave structure over $x$ coordinate}

The waveguide is supposed to be open over the wide transverse coordinate $x$ [Fig. 1(a)]. Let us describe formation of wave structure over this coordinate. For simplicity we assume here that the field structure is fixed over the $y$ coordinate and close to TEM mode. As far as the lengths of Bragg mirrors $l_{1,2}$ are sufficiently smaller than the distance $l_{0}$ between them, we also assume these mirrors to be lumped and characterized by the reflection coefficients $R_{1,2}$. The boundary effects associated with finiteness of mirrors apertures are neglected assuming that the field localization is defined by the optical guiding properties [10-12] of the sheet electron beam possessing the finite width $l_{x}$. In this case the electromagnetic field inside the resonator can be presented as a sum of two counterpropagating quasioptical beams specified by vector potentials:

$$
\vec{A}=\vec{y}_{0} \operatorname{Re}\left\{A_{n}^{+}(z, x) e^{i(\omega t-k z)}+A_{n}^{-}(z, x) e^{i(\omega t+k z)}\right\} .
$$

Taking into account the diffraction effects over the $x$ axis, the process of amplification of the synchronous wave beam $A^{+}$by the electrons on the one passage can be described by the equation [12]:

$$
\frac{i}{2 k} \frac{\partial^{2} \hat{A}_{n}^{+}}{\partial x^{2}}+\frac{\partial \hat{A}_{n}^{+}}{\partial z}=\chi(x) \frac{e I_{0}}{2 m c^{3} \gamma_{0}} \frac{\lambda}{b} K J,
$$

where $n$ is the passage number, $\hat{A}_{n}^{+}=i e A_{n}^{+} / \sqrt{2} m c^{2} \gamma_{0}$. At the large values of the Fresnel parameter

$$
\frac{l_{x}^{2}}{l_{0} \lambda} \gg 1
$$

the diffraction divergence on the one passage is small. In the considered model of high- $Q$ two-mirror cavity the reflection coefficients are close to unity $R_{1,2} \approx 1$ and the amplitude change over the one passage is also small:

$$
\left|\frac{A_{n+1}-A_{n}}{A_{n}}\right| \ll 1 .
$$

The condition (27) and (28) allows one to introduce the differential operators over the "slow" time (see [12] for details) 


$$
\tau=n \frac{2 \omega l_{0}}{c}
$$

Correspondingly, the field evolution inside resonator can be described by equations

$\frac{i}{2 k^{2}} \frac{\partial^{2} \hat{A}}{\partial x^{2}}+\frac{\partial \hat{A}}{\partial \tau}+\frac{1}{2 Q} \hat{A}=\chi(x) \frac{e I_{0}}{4 \pi m c^{3} \gamma_{0}} \frac{\lambda^{2}}{b l_{z}} K \int_{0}^{l_{0}} J d z$,

where $Q=\omega l_{0} / c\left(1-R_{1} R_{2}\right)$ is the quality factor. Introducing the normalized variables and parameters

$$
\begin{aligned}
\hat{\tau} & =\tau / 2 Q, \quad \hat{X}=k x / \sqrt{Q}, \quad \hat{Z}=k z G, \quad \hat{a}=\mu K \hat{A} G^{-2}, \\
G & =\left(\frac{e I_{0}}{m c^{3}} \frac{\mu K^{2}}{2 \pi \gamma_{0}\left(1-R_{1} R_{2}\right)} \frac{\lambda^{2}}{b}\right)^{1 / 3},
\end{aligned}
$$

we obtain a self-consistent system of equations describing the transverse field structure evolution in an FEL with a high- $Q$ two-mirror cavity [8]:

$$
\begin{gathered}
i \frac{\partial^{2} \hat{a}}{\partial \hat{X}^{2}}+\frac{\partial \hat{a}}{\partial \hat{\tau}}+\hat{a}=\chi(\hat{X}) \int_{0}^{\hat{L}_{0}} J d Z \quad \frac{\partial^{2} \theta}{\partial \hat{Z}^{2}}=\operatorname{Re}\left(\hat{a} e^{i \theta}\right) \theta \\
\left.\right|_{\hat{Z}=0}=\theta_{0} \in[0,2 \pi],\left.\frac{\partial \theta}{\partial \hat{Z}}\right|_{\hat{Z}=0}=\hat{\Delta} .
\end{gathered}
$$

Here $\hat{L}_{0}=k l_{0} G$ is the normalized distance between mirrors, $\hat{L}_{x}=k l_{x} / \sqrt{Q}$ is the normalized width of an electron beam, $\hat{\Delta}=\Delta / k G, \chi(\hat{X})$ describes the distribution of the electron current over the $x$ coordinate. On the edges of an electron beam $\hat{X}=0, \hat{L}_{x}$, one should put the radiation boundary conditions which are similar to (20):

$$
\left.a\left(\hat{X}=0, \hat{L}_{x}\right) \mp \frac{1}{\sqrt{\pi i}} \int_{0}^{\hat{\tau}} \frac{e^{\tau^{\prime}-\hat{\tau}}}{\sqrt{\hat{\tau}-\tau^{\prime}}} \frac{\partial a\left(\hat{X}, \tau^{\prime}\right)}{\partial \hat{X}}\right|_{\hat{X}=0, \hat{L}_{x}} d \tau^{\prime}=0 .
$$

Diffraction power losses and the output power are defined correspondingly:

$$
\begin{aligned}
& P_{\mathrm{dif}}^{x}=\operatorname{Im}\left(\left.\hat{a} \frac{\partial \hat{a}^{*}}{\partial \hat{X}}\right|_{\hat{X}=0}+\left.\hat{a}^{*} \frac{\partial \hat{a}}{\partial \hat{X}}\right|_{\hat{X}=\hat{L}_{x}}\right), \\
& P_{\mathrm{rad}}=\int_{0}^{\hat{L}_{x}}|\hat{a}|^{2} d X^{\prime} .
\end{aligned}
$$

We simulate the transverse field structure formation based on Eqs. (28) for the same parameters of undulator and electron beam as used previously in Sec. II A. For mirror reflection coefficients $R_{1,2}=0.6$ the parameter $G$ given by (31) is in order of $2 \times 10^{-4}$ that corresponds to the normalized length $\hat{L}_{0} \approx 7$. At the width of the electron beam of $4 \mathrm{~cm}$ the normalized width is estimated as $\hat{L}_{x} \approx 3$. The results of simulation presented in Fig. 7 demonstrate the formation of the transverse (over $x$ axis) field structure during the establishment of a steady-state generation regime. One can see that in the parameter domain under consideration the spatial structure of the output radiation is regular and localized in the area of the electron beam.

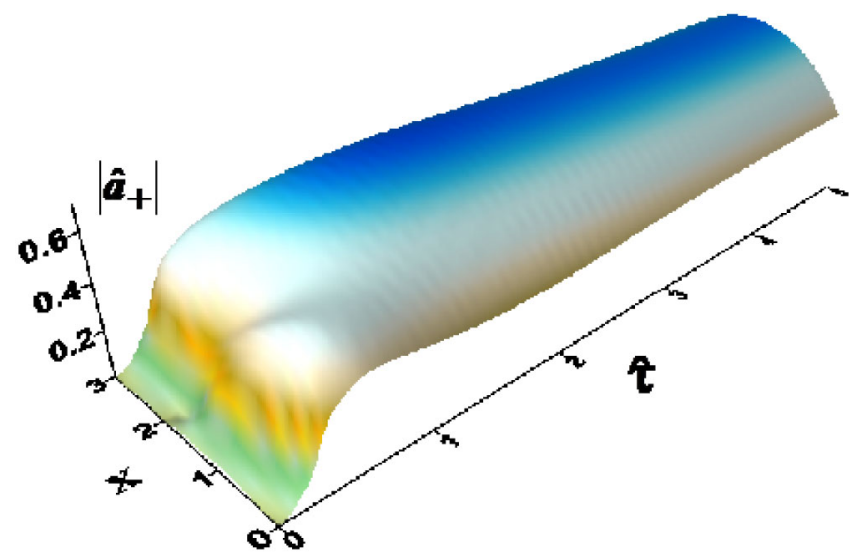

FIG. 7. Formation of the transverse (over $x$ axis) field structure in the process of establishment of the steady-state regime $\left(\hat{L}_{x}=\right.$ $\left.3, \hat{\Delta}=-0.5, \hat{L}_{0}=7\right)$.

The output power $P_{\text {rad }}$ being extracted in the longitudinal direction amounts to $90 \%$ of the total radiation power. This means that the electron beam effectively guides the radiation. Note that estimated in Sec. II A Ohmic losses in the waveguide walls for the simulated THz FEL at the chosen parameters surpass the diffraction losses $P_{\mathrm{dif}}^{x}$ and should be a more important factor for consideration in the final design.

Note that our present investigation of the spatial structure formation along the $x$ coordinate agrees with linear analysis of diffraction mode selection given in [12]. This selection is effective while the corresponding Fresnel parameter satisfies the condition

$$
N_{F}=l_{x}^{2} / l_{\mathrm{eff}} \lambda \leq 1,
$$

where $l_{\text {eff }}=l_{0} /\left(1-R_{1} R_{2}\right)$ is the effective length of wave propagation.

\section{FEL WITH ADVANCED UPSTREAM AND TWO-DIMENSIONAL DOWNSTREAM BRAGG REFLECTORS}

For the width of the system exceeding the limits specified by condition (35), the transverse synchronization of radiation can be obtained by using a 2D Bragg structure $[8,9]$ as a downstream mirror [see Fig. 1(b)]. As in Sec. II B we assume that the size of the system in the $y$ direction is limited by the condition $b_{0} \leq 10 \lambda$ that allows one to consider the field structures over this axis fixed and close to TEM mode. Under such an assumption the field in the 2D Bragg structure can be presented as the sum of four wave beams:

$$
\begin{aligned}
\vec{H}= & \vec{x}_{0} \operatorname{Re} \mid\left[A_{+}(x, z, t) e^{-i h z}+A_{-}(x, z, t) e^{i h z}\right. \\
& \left.\left.+G_{+}(x, z, t) e^{-i h x}+G_{-}(x, z, t) e^{i h x}\right] e^{i \omega t}\right] .
\end{aligned}
$$

A specific feature of 2D Bragg structure is the mutual coupling of $A_{ \pm}$partial waves, propagating along $\pm z$ 
directions, with $G_{ \pm}$waves, propagating in the perpendicular $\pm x$ directions. This coupling can be described by the following equations:

$$
\begin{array}{r}
\frac{\partial a_{+}}{\partial Z}+\frac{\partial a_{+}}{\partial \tau}+i \alpha_{2 D}\left(g_{+}+g_{-}\right)=J \\
-\frac{\partial a_{-}}{\partial Z}+\frac{\partial a_{-}}{\partial \tau}+i \alpha_{2 D}\left(g_{+}+g_{-}\right)=0 \\
\pm \frac{\partial g_{ \pm}}{\partial X}+\frac{\partial g_{ \pm}}{\partial \tau}+i \alpha_{2 D}\left(a_{+}+a_{-}\right)=0,
\end{array}
$$

where the coupling coefficient is

$$
\alpha_{2 \mathrm{D}}=b_{2 \mathrm{D}} / 4 b_{0} C .
$$

Two additional wave beams $G_{ \pm}$propagating in the transverse $\pm x$ directions provide synchronization of radiation of the large-size sheet electron beam. Similarly to (21) we

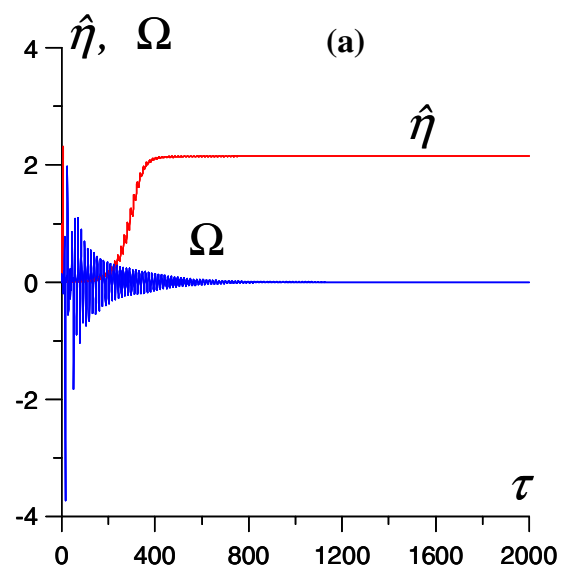

(b)

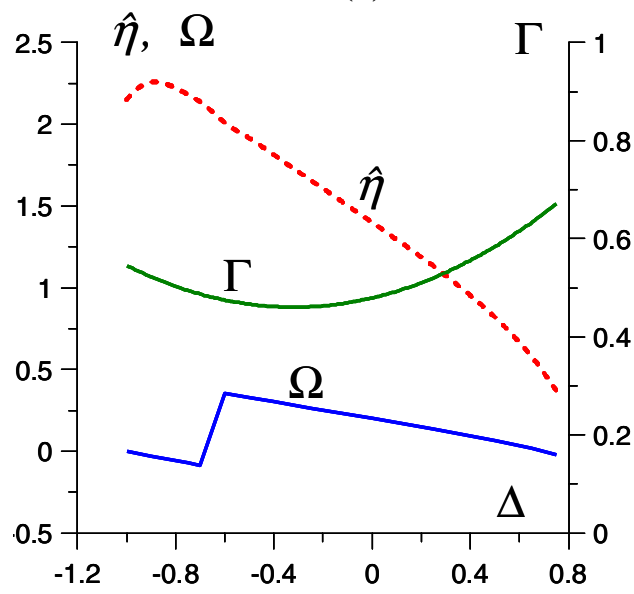

FIG. 8. Modeling of FEL with advanced upstream and 2D downstream Bragg reflectors. Establishment of the steady-state oscillation regime: temporal dependence of normalized efficiency $\hat{\eta}$ and frequency shift $\Omega$ at $\Delta=-0.5$ (a); normalized efficiency $\hat{\eta}$, the electron frequency shift $\Omega$, and the factor $\Gamma$ vs electron synchronism detuning parameter $\Delta$ in the steady-state regime $\left(L_{1}=1, L_{0}=5.5, L_{2}=0.8, \alpha_{1 \mathrm{D}}=0.7, \alpha_{2 \mathrm{D}}=0.5\right.$, $\left.\sigma=0.2, C \approx 1.4 \times 10^{-4}\right)(\mathrm{b})$. apply the boundary conditions that correspond to the absence of external energy fluxes on the edges of the 2D Bragg structure:

$$
\left.a_{-}\right|_{Z=L}=0,\left.\quad g_{+}\right|_{X=0}=0,\left.\quad g_{-}\right|_{X=L_{x}}=0 .
$$

We simulate the nonlinear dynamics of an FEL described by Eqs. (19) and (37) at the same operating frequency $f=1 \mathrm{THz}$ and for the same beam and undulator parameters as in Sec. II that correspond to the gain parameter $C \approx 1.4 \times 10^{-4}$. Lengths of the input and the output Bragg reflectors were taken $l_{1}=35 \mathrm{~cm}$ and $l_{2}=28 \mathrm{~cm}$, corrugation depths $1.2 \mu \mathrm{m}$ and $2 \mu \mathrm{m}$ $\left(L_{1}=1, L_{2}=0.8, \alpha_{1 \mathrm{D}}=0.7, \alpha_{2 \mathrm{D}}=0.5\right)$. The length of regular section was taken $l_{0}=195 \mathrm{~cm}\left(L_{0}=5.5\right)$, the gap between plates was limited by $b_{0} \sim 10 \lambda$. The simulated width of the resonator and the sheet electron beam $\sim 35 \mathrm{~cm}$ $(l \sim 100 \lambda)$ corresponds to normalized width $L_{x}=1$.

Figure 8(a) shows the process of establishment of the steady-state generation regime. Distribution of partial wave amplitudes in this regime (Fig. 9) demonstrates the spatial synchronization of radiation from a sheet electron

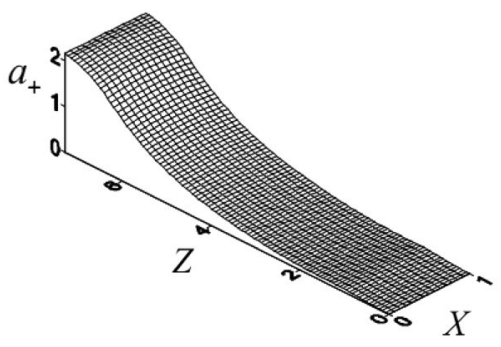

(a)

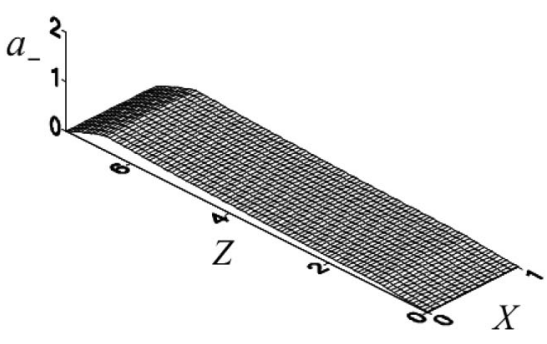

(b)

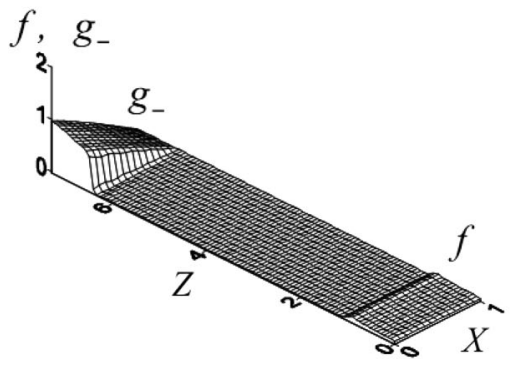

(c)

FIG. 9. Spatial distributions of partial wave beams in the steadystate regime for the same parameters as in Fig. 8: (a),(b), the propagating wave amplitudes $a_{ \pm}(Z, X)$, (c) the cutoff mode $f(Z, X)$ in the upstream advanced Bragg reflector and one of the "transverse synchronization" waves $g_{-}(Z, X)$ in the downstream 2D Bragg reflector. 
beam at the specified parameters. As for the FEL scheme considered in Sec. II, the main amplification of radiation takes place in the regular section of the waveguide after the input advanced Bragg mirror. The output 2D Bragg mirror provides a relatively small (about $15 \%$ power) reflection in the backward wave $a_{-}$. In this case the scattering into the "synchronization waves" $g_{ \pm}$which deflect the radiation energy in the transverse $\pm x$ directions does not exceed $20 \%$ power. So the main part of the power radiated by the electron beam is extracted with the forward propagating wave $a_{+}$. In the variant under consideration, the factor $\Gamma$ amounts to $60 \%$. Correspondingly, at the electron efficiency $\eta \sim 2 \%$ the gross radiation power reaches $\sim 350 \mathrm{MW}$.

To conclude, we note that in contrast to the existing terahertz FEL based on linear rf accelerators [20,21] and microtrons [22] whose beams are sequences of short (picosecond) electron pulses, the proposed scheme can be used to create generators based on intense quasicontinuous (microsecond) electron beams formed by linear induction accelerators [23] or electrostatic accelerators [24,25], where the mirrors of the FEL cavity should be compatible with the beam transport channel. Correspondingly, an advantage of a new scheme is the possibility of reaching a high (multimegawatt) average power of terahertz radiation and high pulse energy of 1-10 J. At the moment we are planning a modification of the linear induction accelerator-LIU 3000 (JINR, Dubna) - to match beam parameters described in this paper.

\section{ACKNOWLEDGMENTS}

The authors are grateful to K. Kamada, A. K. Kaminsky, and S.N. Sedykh for useful discussions. This work is partially supported by the Russian Foundation for Basic Research (Grant No. 10-08-01269) and the Russian President grant for young Ph.D. scientists (Grant No. MK-5530.2011.2).

[1] H. Kogelnik and C. V. Shank, J. Appl. Phys. 43, 2327 (1972).

[2] A. Yariv, Quantum Electronics (Wiley, New York, 1975).

[3] V. L. Bratman, G. G. Denisov, N. S. Ginzburg, and M. I. Petelin, IEEE J. Quantum Electron. 19, 282 (1983).
[4] N.S. Ginzburg, A. A. Kaminsky, A. K. Kaminsky, N. Yu. Peskov, S. N. Sedykh, A. P. Sergeev, and A.S. Sergeev, Phys. Rev. Lett. 84, 3574 (2000).

[5] N.S. Ginzburg, A. M. Malkin, A. A. Kaminsky, A. K. Kaminsky, N. Yu. Peskov, S. N. Sedykh, A. P. Sergeev, and A.S. Sergeev, Phys. Rev. ST Accel. Beams 8, 040705 (2005).

[6] N.S. Ginzburg, A.M. Malkin, N. Yu. Peskov, A. S. Sergeev, V. Yu. Zaslavsky, K. Kamada, and R. Ando, Phys. Rev. ST Accel. Beams 12, 060702 (2009).

[7] N.S. Ginzburg, A.M. Malkin, N. Yu. Peskov, A. S. Sergeev, V. Yu. Zaslavsky, K. Kamada, and R. Ando, Appl. Phys. Lett. 95, 043504 (2009).

[8] N.S. Ginzburg, N. Yu. Peskov, and A. S. Sergeev, Opt. Commun. 96, 254 (1993).

[9] N.S. Ginzburg, N. Yu. Peskov, A. S. Sergeev, A. D. R. Phelps, I. V. Konoplev, G. R. M. Robb, A. W. Cross, A. V. Arzhannikov, and S. L. Sinitsky, Phys. Rev. E 60, 935 (1999).

[10] E. T. Scharlemann, A. M. Sessler, and J. S. Wurtele, Phys. Rev. Lett. 54, 1925 (1985).

[11] G. T. Moore, Opt. Commun. 52, 46 (1984).

[12] N. S. Ginzburg, N.F. Kovalev, and N. Yu. Rusov, Opt. Commun. 46, 300 (1983).

[13] A. V. Gaponov et al., ZETP Lett. 2, 430 (1965) [in Russian].

[14] F. S. Rusin and G. D. Bogomolov, ZETP Lett. 4, 236 (1966) [in Russian].

[15] N. S. Ginzburg, V. Yu. Zaslavsky, I. V. Zotova, A. M. Malkin, N. Yu. Peskov, and A. S. Sergeev, JETP Lett. 91, 266 (2010).

[16] B.Z. Katsenelenbaum, Theory of Irregular Waveguides with Slowly Varying Parameters (Akademie Nauk SSSR, Moscow, 1961).

[17] M. I. Fuks et al., IEEE Trans. Plasma Sci. 32, 1323 (2004).

[18] N. S. Ginzburg, N. A. Zavolsky, G. S. Nusinovich, and A. S. Sergeev, Sov. Radiophys. Quantum Electron. 29, 89 (1986).

[19] N. S. Ginzburg, G. S. Nusinovich, and N. A. Zavolsky, Int. J. Electron. 61, 881 (1986).

[20] M. A. Dem'yanenko et al., Appl. Phys. Lett. 92, 131116 (2008).

[21] G. R. Neil et al., Phys. Rev. Lett. 84, 662 (2000).

[22] G. M. Kazakevich et al., Phys. Rev. ST Accel. Beams 12, 040701 (2009).

[23] T. J. Orzechowski et al., Phys. Rev. Lett. 57, 2172 (1986).

[24] L. R. Elias, G. Ramian, J. Hu, and A. Amir, Phys. Rev. Lett. 57, 424 (1986).

[25] A. Abramovich, Phys. Rev. Lett. 82, 5257 (1999). 